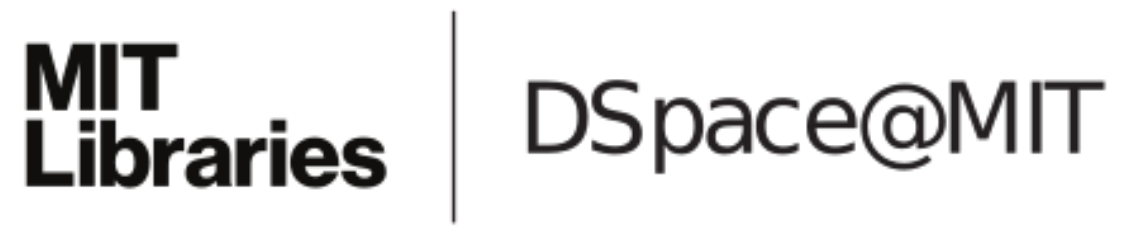

\author{
MIT Open Access Articles
}

\section{Efficient Conservative Numerical Schemes for 1D Nonlinear Spherical Diffusion Equations with Applications in Battery Modeling}

The MIT Faculty has made this article openly available. Please share how this access benefits you. Your story matters.

Citation: Zeng, Y., P. Albertus, R. Klein, N. Chaturvedi, A. Kojic, M. Z. Bazant, and J. Christensen. “Efficient Conservative Numerical Schemes for 1D Nonlinear Spherical Diffusion Equations with Applications in Battery Modeling." Journal of the Electrochemical Society 160, no. 9 (January 1, 2013): A1565-A1571. (C) 2013 The Electrochemical Society.

As Published: http://dx.doi.org/10.1149/2.102309jes

Persistent URL: http://hdl.handle.net/1721.1/91234

Version: Final published version: final published article, as it appeared in a journal, conference proceedings, or other formally published context

Terms of Use: Article is made available in accordance with the publisher's policy and may be subject to US copyright law. Please refer to the publisher's site for terms of use. 


\title{
(10) \\ Efficient Conservative Numerical Schemes for 1D Nonlinear Spherical Diffusion Equations with Applications in Battery Modeling
}

\author{
Yi Zeng, ${ }^{\text {a,z }}$ Paul Albertus, ${ }^{\text {b,* }}$ Reinhardt Klein,, ${ }^{\text {b Nalin Chaturvedi, }{ }^{b} \text { Aleksandar Kojic, }}$ \\ Martin Z. Bazant, ${ }^{\text {,ac,**}}$ and Jake Christensen ${ }^{\mathrm{b}, *}$ \\ ${ }^{a}$ Department of Mathematics, Massachusetts Institute of Technology, Cambridge, Massachusetts 02138, USA \\ ${ }^{b}$ Robert Bosch Research and Technology Center, Palo Alto, California 94304, USA \\ ${ }^{c}$ Department of Chemical Engineering, Massachusetts Institute of Technology, Cambridge, Massachusetts 02138, USA
}

\begin{abstract}
Mathematical models of batteries which make use of the intercalation of a species into a solid phase need to solve the corresponding mass transfer problem. Because solving this equation can significantly add to the computational cost of a model, various methods have been devised to reduce the computational time. In this paper we focus on a comparison of the formulation, accuracy, and order of the accuracy for two numerical methods of solving the spherical diffusion problem with a constant or non-constant diffusion coefficient: the finite volume method and the control volume method. Both methods provide perfect mass conservation and second order accuracy in mesh spacing, but the control volume method provides the surface concentration directly, has a higher accuracy for a given numbers of mesh points and can also be easily extended to variable mesh spacing. Variable mesh spacing can significantly reduce the number of points that are required to achieve a given degree of accuracy in the surface concentration (which is typically coupled to the other battery equations) by locating more points where the concentration gradients are highest.

(C) 2013 The Electrochemical Society. [DOI: 10.1149/2.102309jes] All rights reserved.
\end{abstract}

Manuscript submitted May 10, 2013; revised manuscript received July 3, 2013. Published July 18, 2013.

The modeling of battery systems in which an atom intercalates into solid particles has received significant attention, ${ }^{1}$ especially with regard to the $\mathrm{Li}$-ion and $\mathrm{Ni} / \mathrm{MH}$ chemistries. ${ }^{2-6}$ A popular way to treat diffusion of an insertion atom into a solid phase while avoiding the solution of a full two-dimensional (2D) problem is to construct a pseudo-2D model, in which one dimension extends between the two current collectors and a second dimension extends into the solid particles, with a coupling between the two dimensions at the surface of the intercalation particles. A schematic of the process of spherical diffusion and coupling at the particle surface is shown in Fig. 1, where we assume that diffusion occurs in the radial direction in an isotropic medium. Although more complex processes for solid intercalation have been proposed for phase-separating active materials, such as $\mathrm{LiFePO}_{4},{ }^{1,7-12}$ here we focus only on the most common approximation of 1D spherical diffusion. This approximation is also invoked to model the diffusion impedance of insertion batteries. ${ }^{13}$

The concentration at the surface of the particle should be obtained accurately because it is used in the exchange current density for the interfacial reaction, where its contribution is typically non-linear $(\mathrm{a} 1 / 2$ power is common) as well as in the calculation of the equilibrium potential for the interfacial reaction, which is also typically nonlinear. ${ }^{5}$ The surface concentration affects the charge-transfer reaction rate and the interfacial potential because it contributes to the activity of the intercalated ions. In a general theory of electrochemical kinetics based on non-equilibrium thermodynamics, ${ }^{1}$ the surface activity is also affected by concentration gradients,,${ }^{9,10}$ elastic coherency strain, ${ }^{11}$ surface "wetting" 12 and other non-idealities in solids. For an isotropic spherical particle, it can be shown that the general Cahn-Hilliard reaction model, ${ }^{1,14}$ which allows for complex thermodynamics with phase separation, reduces to the simple model considered here - spherical diffusion with concentration-dependent kinetics - in the case of a solid-solution material, whose equilibrium state is homogeneous. ${ }^{15}$

Several different methods have been employed for the solution of the $1 \mathrm{D}$ spherical diffusion problem, including Duhamel's superposition integral, ${ }^{3}$ diffusion length method, ${ }^{16}$ polynomial approximation, ${ }^{17}$ PSS method, ${ }^{18,19}$ penetration depth method, ${ }^{20}$ finite element method, ${ }^{20}$ finite difference method, ${ }^{21}$ and finite volume method. Some methods are only valid under certain circumstance, for instance, Duhamel's superposition integral can only handle the linear problem, requiring the use of a constant diffusion coefficient. In practice solid-phase diffusion coefficients often depend on both composition (i.e., local

${ }^{*}$ Electrochemical Society Active Member.

${ }^{\mathrm{z} E}$-mail: yizeng@math.mit.edu
Li concentration) and temperature, so the ability to solve the case in which the diffusion coefficient can vary is very important. A review of existing methods for solving solid diffusion methods in terms of their numerical performance and restrictions is given by Subramanian. ${ }^{21}$

Because each node point within the electrode is coupled to the 1D spherical diffusion problem, the total number of spatially distributed variables to be solved at each timestep(i.e., states) in the model can be increased dramatically by the inclusion of a finely meshed radial spatial dimension. For example, with 50 node points in each electrode (and 20 in the separator), and six equations in the cell sandwich dimension, adding a spherical diffusion dimension with 50 nodes points would increase the number of states in the model from 720 to 5720 . This was the original reason for the use of Duhamel's superposition integral (no additional states are added in the particle dimension), ${ }^{3}$ and is the reason that significant work has been invested to find efficient computational solutions to the non-linear 1D spherical diffusion problem.

Among the numerical methods that are suitable for solving the solid diffusion problem with a variable diffusion coefficient, the finite volume method is well known for its perfect mass conservation. This property is a great advantage in long term simulations in which a

\section{Spherical insertion particle}

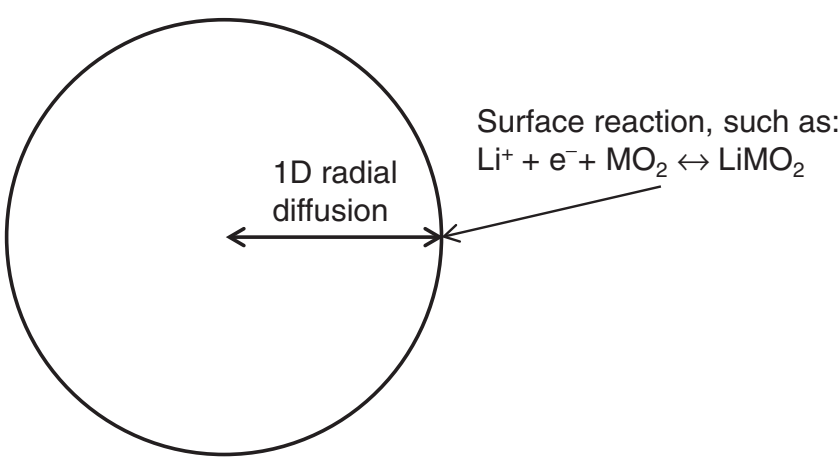

Figure 1. Schematic of the physical model addressed in this paper. An electrochemical surface reaction (e.g., involving the insertion and removal of $\mathrm{Li}$ from a metal oxide ( $\mathrm{M}=\mathrm{Co}, \mathrm{Ni}, \mathrm{Mn}$, or others) $)$ supplies a specified flux of $\mathrm{Li}$ at the surface of a spherical particle in which radial, one-dimensional, Fickian diffusion takes place. 
gain or loss of mass can significantly influence simulation results. However, the finite volume method may not be as computationally efficient as other methods such as the finite difference or finite element method. In this paper, we will present another conservative numerical scheme, the control volume method, which is both computationally efficient and simple to implement. To the best of our knowledge, this method and its extension to non-uniform mesh spacing has not been previously published for the spherical diffusion problem, and this work is therefore an important advance in the ability to solve the spherical diffusion problem with a diffusion coefficient that depends on composition, temperature, or other factors, in a conservative and efficient manner.

We can formulate the process of intercalation of a species into a spherical solid particle with the 1D spherical diffusion equation and the Neumann condition at the particle surface,

$$
r^{2} \frac{\partial c}{\partial t}=\frac{\partial}{\partial r}\left(D r^{2} \frac{\partial c}{\partial r}\right),
$$

while the boundary conditions are,

$$
\begin{aligned}
\left.\frac{\partial c}{\partial r}\right|_{(r=0)} & =0, \\
\left.D \frac{\partial c}{\partial r}\right|_{\left(r=R_{s}\right)} & =-j(t) .
\end{aligned}
$$

The diffusion coefficient may be a function of concentration and spatial position, as well as a function of temperature $T$ and other quantities. All parameters in the partial differential equations system in Eq. 1 are with SI units.

In this work we set the diffusion coefficient to be a function of concentration alone, and use a fit to measurements done on $\mathrm{Li}\left(\mathrm{Ni}_{1 / 3} \mathrm{Mn}_{1 / 3} \mathrm{Co}_{1 / 3}\right) \mathrm{O}_{2},{ }^{22}$

$$
\begin{aligned}
& D(c)=D^{r e f}\left(1+100(S O C)^{\frac{3}{2}}\right), \\
& S O C=\frac{c^{\max }-c}{c^{\max }} \frac{\hat{C}_{\text {theory }}}{\hat{C}_{\text {practical }}} .
\end{aligned}
$$

The values of the parameters used in Eq. 4 are given in Table I.

A plot of the function given by Eq. 4 as well as the original measured data obtained by Wu et al. ${ }^{22}$ are given in Fig. 2 .

\section{The Finite Volume and Control Volume Formulations}

Before turning to the control volume method, for the sake of comparison we first consider the finite volume method, which is a well-developed numerical discretization method for partial differential equations, especially in the transport problem. The finite volume method is well known for its robustness and efficiency in computations, and most importantly, for its perfect mass conservation. In this section, we first introduce the spatial discretization by the finite volume method, and next analyze the error order of this method.

The finite volume Method.-Formulation of the finite volume method.- The basic idea for the finite volume method is to solve for the integral form of the original PDE. We assume the diffusion coefficient function $D(c)$ is globally Lipschitz, ${ }^{23}$ then integrate both

Table I. Parameter settings for the diffusion coefficient function in Eq. 4.

\begin{tabular}{lccc}
\multicolumn{1}{c}{ Parameter Name } & Notation & Value & Unit \\
\hline Reference Diffusivity & $D^{\text {ref }}$ & $2.00 \times 10^{-16}$ & $\mathrm{~m}^{2} / \mathrm{s}$ \\
Maximum Concentration & $C^{\text {max }}$ & $4.665 \times 10^{4}$ & $\mathrm{~mol} / \mathrm{m}^{3}$ \\
Theoretical Capacity & $\hat{C}_{\text {theory }}$ & 277.84 & $\mathrm{mAh} / \mathrm{g}$ \\
Practical Capacity & $\hat{C}_{\text {practical }}$ & 160 & $\mathrm{mAh} / \mathrm{g}$
\end{tabular}

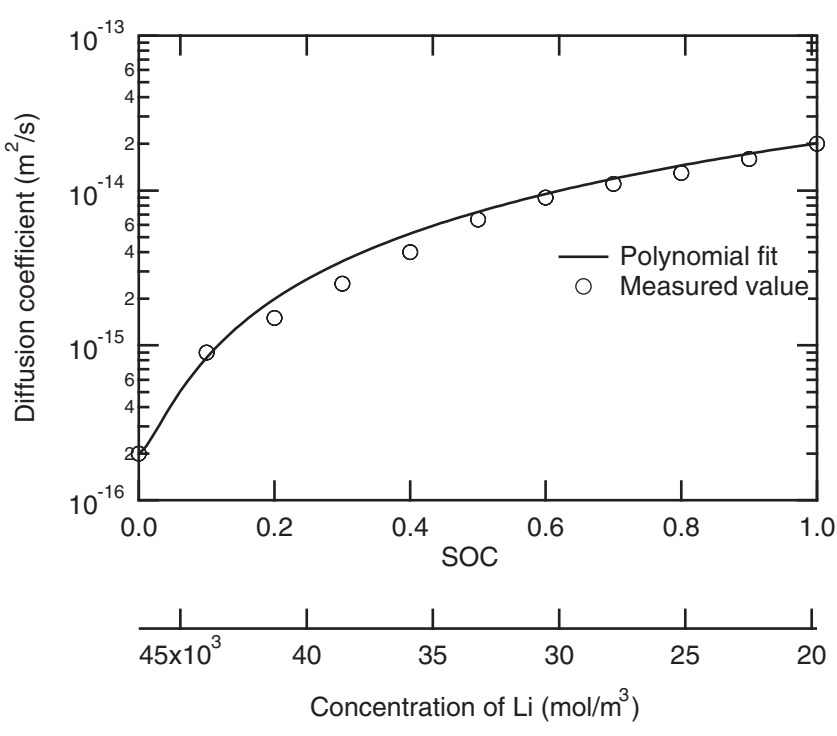

Figure 2. Measured values of the lithium diffusion coefficient in $\mathrm{Li}\left(\mathrm{Ni}_{1 / 3} \mathrm{Mn}_{1 / 3} \mathrm{Co}_{1 / 3}\right) \mathrm{O}_{2}$ (circles) and the fitting polynomial function in Eq. 4 (curve) we use for the numerical simulation.

sides of Eq. 1 over the interval $\left[r_{i}, r_{i+1}\right]$, to get,

$$
\begin{aligned}
\frac{\partial \bar{c}_{i}}{\partial t} V_{i}= & D\left(\frac{\bar{c}_{i+1}+\bar{c}_{i}}{2}\right) r_{i+1}^{2} \frac{\bar{c}_{i+1}-\bar{c}_{i}}{\Delta r} \\
& -D\left(\frac{\bar{c}_{i}+\bar{c}_{i-1}}{2}\right) r_{i}^{2} \frac{\bar{c}_{i}-\bar{c}_{i-1}}{\Delta r}+O\left(\Delta r^{2}\right),
\end{aligned}
$$

where $V_{i}=\frac{1}{3}\left(r_{i+1}^{3}-r_{i}^{3}\right)$ is the scaled volume of the shell between $\left[r_{i}, r_{i+1}\right]$, and $\bar{c}_{i}$ is the average concentration within this small volume. Since the volume $\frac{4}{3} \pi\left(r_{i+1}^{3}-r_{i}^{3}\right)$ is always canceled with the surface area $4 \pi r_{i}^{2}$ by the same factor $4 \pi$, we will by default use this scaled volume $V_{i}=\frac{1}{3}\left(r_{i+1}^{3}-r_{i}^{3}\right)$ and scaled surface area $r_{i}^{2}$ without further notation. Eq. 5 holds for each shell, and in fact it gives the spatial discretization of our original PDE system given by Eq. 1 .

We may also write each of the above discretized equations in the following matrix form,

$$
\mathbf{M}_{f} \frac{\partial \overline{\mathbf{c}}}{\partial t}=\mathbf{f}(\overline{\mathbf{c}}),
$$

where $\mathbf{M}_{f}$ is a mass matrix with the volume of each shell on its diagonal and zero elsewhere, $\mathbf{f}$ is the vector function with each item given by the left hand side of Eq. 5, and $\overline{\mathbf{c}}$ is the vector of average concentration in each shell.

A disadvantage of this method for use in intercalation battery models is that it computes volume-averaged concentrations rather than concentrations spatially located at the node points. Thus, in order to obtain the surface concentration, which determines the equilibrium potential that goes into the exponential of the kinetic expression and is typically part of the exchange-current density, it is necessary to extrapolate the concentration at the particle surface by,

$$
c_{\text {surface }}=\frac{3 \bar{c}_{N}-\bar{c}_{N-1}}{2} \text {. }
$$

Error order analysis in spatial coordinates for the finite volume method.- In our system, we will demonstrate that this discretization method in fact achieves second order accuracy in the spatial coordinate. We also show that the surface concentration converges in second order in mesh spacing. To demonstrate this point, instead of proving directly that this finite volume discretization method is in second order, we will derive another second order accurate method and show these two methods are equivalent. 
Let $c_{i}$ be the midpoint concentration of the interval $\left[r_{i}, r_{i+1}\right]$ instead of the average concentration within this shell. Then by Taylor expansion, we can easily get,

$$
\begin{aligned}
\frac{\partial}{\partial t}\left(V_{i} c_{i}+O\left(\Delta r^{3}\right)\right) & \\
= & \int_{r_{i}}^{r_{i+1}} r^{2} \frac{\partial c}{\partial t} d r=\int_{r_{i}}^{r_{i+1}} \frac{\partial}{\partial r}\left(D r^{2} \frac{\partial c}{\partial r}\right) d r \\
= & D\left(\frac{c_{i+1}+c_{i}}{2}\right) r_{i+1}^{2} \frac{c_{i+1}-c_{i}}{\Delta r} \\
& -D\left(\frac{c_{i}+c_{i-1}}{2}\right) r_{i}^{2} \frac{c_{i}-c_{i-1}}{\Delta r}+O\left(\Delta r^{2}\right) .
\end{aligned}
$$

Since this derivation is valid on each interval, the overall error of the above discretization method is of second order, $O\left(\Delta r^{2}\right)$, for both sides of Eq. 1. Thus, this method is of second order accurate in space as desired.

Given that $c_{N-1}$ and $c_{N}$ are second order accurate, the extrapolation of $c_{\text {surface }}=\frac{2 \bar{c}_{N}-\bar{c}_{N-1}}{2}$ is then obviously second order accurate by Taylor's expansion.

Not surprisingly, Eq. 8 is in exactly the same form as Eq. 5, if we neglect the error term. Therefore, these two discretization methods are equivalent. This finishes our proof that the error of finite volume discretization in Eq. 6 should converge in second order in the spatial coordinate.

Instead of only using the average concentrations of the last two intervals to approach the surface concentration as proposed by Eq. 7, we may use the average concentration from all $\mathrm{N}$ intervals to achieve a more accurate surface concentration. Let $c_{\text {left }}$ be the nodal concentration on the left boundary of an interval $\left[r_{i}, r_{i+1}\right]$, and $c_{\text {right }}$ be the concentration on the right node of the corresponding interval, then by Taylor's expansion we have,

$$
\bar{c}_{i}=\frac{c_{\text {left }}+c_{\text {right }}}{2}+\frac{c_{\text {left }}-c_{\text {right }}}{6} \frac{\Delta r}{r_{i+1}}+O\left(\Delta r^{2}\right) .
$$

Since we have $N$ intervals in total, it gives us $N$ constraints. Yet we have $N+1$ unknown variables of nodal concentration, we may also use two Neumann boundary conditions. Then by solving the least squares problem we can obtain the concentration on each node, including the surface concentration. This may help us to gain a more accurate surface concentration, but still in second order convergence. The effect of this method will be shown in the numerical experiment section.

The control volume method.- We have discussed several advantages for using the finite volume method to discretize our PDE system in the previous section, yet this method may not be ideal for our specific spherical diffusion problem in a model of an intercalation battery. We are essentially interested in the concentration at the surface of the particle, which is the quantity that is coupled into the full set of battery model equations, but as mentioned above, the finite volume method does not immediately provide this information. The extrapolation step described above may take additional computation effort and introduce new numerical error. Therefore, here we show the development of a new numerical algorithm that keeps the advantages of the finite volume method while avoiding the extrapolation step.

With this motivation, in this section we derive a new numerical discretization method for our spherical diffusion equation, which we call the control volume method. We will first introduce a basic version of this method, and provide the theoretical proof of the error convergence order. Then a modified version is shown for better mass conservation purpose, together with a discussion of its accuracy order. We also show the generalization to a non-uniform grid mesh of this modified control volume method. While the finite-volume method can be used with variable mesh spacing, the extension of the control-volume method to variable mesh spacing is more straightforward.
Derivation of the control volume method.-The control volume method is also a numerical scheme that discretizes the PDE according to the integral form of Eq. 1 . We now mesh the spatial domain $\left[0, R_{p}\right]$ uniformly with $N$ points, denoted as $r_{1}, r_{2}, \cdots, r_{N}$, while $r_{1}=0$ and $r_{N}=R_{p}$. For our convenience, we define $\Delta r$ to be the distance between two nearby mesh points. We denote $r_{i}+\frac{1}{2} \Delta r$ as $r_{i+\frac{1}{2}}$ and $r_{i}-\frac{1}{2} \Delta r$ as $r_{i-\frac{1}{2}}$.

If we integrate the left hand side of the equation over an interval centered at $r_{i}(i \neq 1,2$ or $N)$ with width $\Delta r,\left[r_{i-\frac{1}{2}}, r_{i+\frac{1}{2}}\right]$, then we get,

$$
\int_{r_{i}-\frac{1}{2} \Delta r}^{r_{i}+\frac{1}{2} \Delta r} r^{2} \frac{\partial c}{\partial t} d r=\frac{\partial}{\partial t}\left(\int_{r_{i}-\frac{1}{2} \Delta r}^{r_{i}} r^{2} c d r+\int_{r_{i}}^{r_{i}+\frac{1}{2} \Delta r} r^{2} c d r\right) .
$$

The function $f(r)=r^{2} c(r)$ takes values $r_{i-1}^{2} c_{i-1}$ at the mesh point $r_{i-1}$ and $r_{i}^{2} c_{i}$ at the point $r_{i}$. Then for any $r$ in the sub-interval $\left[r_{i-\frac{1}{2}}, r_{i}\right]$, by Taylor's expansion, we can approximate the value of function $f(x)$ by the following equation,

$$
f(r)=r_{i}^{2} c_{i}+\left(r-r_{i}\right) \frac{r_{i}^{2} c_{i}-r_{i-1}^{2} c_{i-1}}{\Delta r}+O\left(\Delta r^{2}\right) .
$$

Similarly, in the sub-interval $\left[r_{i}, r_{i+\frac{1}{2}}\right]$, we apply the same technique and get,

$$
f(r)=r_{i}^{2} c_{i}+\left(r-r_{i}\right) \frac{r_{i+1}^{2} c_{i+1}-r_{i}^{2} c_{i}}{\Delta r}+O\left(\Delta r^{2}\right) .
$$

Putting these two formulae back into Eq. 10, we get,

$$
\begin{aligned}
& \int_{r_{i}-\frac{1}{2} \Delta r}^{r_{i}+\frac{1}{2} \Delta r} r^{2} \frac{\partial c}{\partial t} d r \\
& \quad=\Delta r \frac{\partial}{\partial t}\left(\frac{1}{8} r_{i+1}^{2} c_{i+1}+\frac{6}{8} r_{i}^{2} c_{i}+\frac{1}{8} r_{i-1}^{2} c_{i-1}+O\left(\Delta r^{2}\right)\right) .
\end{aligned}
$$

If we also integrate the right hand side of Eq. 1 as we did for the finite volume method, and equate the two sides, we obtain,

$$
\begin{aligned}
\Delta r & \frac{\partial}{\partial t}\left(\frac{1}{8} r_{i-1}^{2} c_{i-1}+\frac{6}{8} r_{i}^{2} c_{i}+\frac{1}{8} r_{i+1}^{2} c_{i+1}+O\left(\Delta r^{2}\right)\right) \\
= & \left(D\left(\frac{c_{i+1}+c_{i}}{2}\right) r_{i+\frac{1}{2}}^{2} \frac{c_{i+1}-c_{i}}{\Delta r}\right. \\
& \left.-D\left(\frac{c_{i}+c_{i-1}}{2}\right) r_{i-\frac{1}{2}}^{2} \frac{c_{i}-c_{i-1}}{\Delta r}+O\left(\Delta r^{2}\right)\right) .
\end{aligned}
$$

Since the boundary condition is the Neumann condition, it can be easily handled in this control volume method in the same way as the finite volume method.

From the error terms above, we see that this method is also second order accurate in the spatial discretization. However, this method has two main problems. First, since we have no information about $c_{1}$ due to $x_{1}=0$, then we have $N-1$ unknown variables but $N$ equations, which shows the system is over-determined. Second, if we sum up Eq. 14 of each interval, we get,

$$
\frac{\partial}{\partial t}\left(\sum_{i=1}^{N-1} r_{i}^{2} c_{i}+\frac{1}{2} r_{N}^{2} c_{N}\right)+j \frac{r_{N}^{2}}{\Delta r}=0 .
$$

If we apply the constant flux for some time period $\Delta t$ then relax the system to make the concentration flat in the entire domain, the concentration increment should be the same in the whole spatial coordinate. However, let $V_{\text {total }}=\lim _{N \rightarrow \infty}\left(\sum_{i=1}^{N-1} r_{i}^{2}+\frac{1}{2} r_{N}^{2}\right) \Delta r=\frac{1}{3} r_{N}^{3}$ be the total particle volume, we have,

$$
V_{\text {total }} \Delta c \neq\left(\sum_{i=1}^{N-1} r_{i}^{2}+\frac{1}{2} r_{N}^{2}\right) \Delta c=-j \frac{r_{N}^{2}}{\Delta r} \Delta t .
$$


The concentration increment is always off from the true value for a certain percentage, which violates the mass conservation law of our system.

Modification for mass conservation.-In order to fix the problems described above, we replace $r_{i}^{2} \Delta r$ by the volume $V_{i}$ of the corresponding shell $\left[r_{i-\frac{1}{2}}, r_{r+\frac{1}{2}}\right]$. For example,

$$
V_{i}=\frac{\left(r_{i}+\frac{\Delta r}{2}\right)^{3}-\left(r_{i}-\frac{\Delta r}{2}\right)^{3}}{3}=r_{i}^{2} \Delta r+\frac{1}{12} \Delta r^{3} .
$$

Keeping the right hand side of Eq. 14 unchanged, we then obtain,

$$
\begin{aligned}
\frac{\partial}{\partial t}( & \left.\frac{1}{8} V_{i-1} c_{i-1}+\frac{6}{8} V_{i} c_{i}+\frac{1}{8} V_{i+1} c_{i+1}+O\left(\Delta r^{3}\right)\right) \\
= & \left(D\left(\frac{c_{i+1}+c_{i}}{2}\right) r_{i+\frac{1}{2}}^{2} \frac{c_{i+1}-c_{i}}{\Delta r}\right. \\
& \left.-D\left(\frac{c_{i}+c_{i-1}}{2}\right) r_{i-\frac{1}{2}}^{2} \frac{c_{i}-c_{i-1}}{\Delta r}+O\left(\Delta r^{2}\right)\right) .
\end{aligned}
$$

Like Eq. 14, this discretization scheme is also accurate to order $\Delta r^{2}$.

We can further write the new Eq. 18 into a matrix form, which is similar to the equation system Eq. 6 ,

$$
\mathbf{M}_{c} \frac{\partial \mathbf{c}}{\partial t}=\mathbf{f}(\mathbf{c})
$$

where $\mathbf{c}$ is the vector of concentration on each node point, and $\mathbf{M}_{c}$ is a tri-diagonal mass matrix as following,

$$
\mathbf{M}_{c}=\left(\begin{array}{cccccccc}
\frac{3}{4} V_{1} & \frac{1}{8} V_{2} & 0 & 0 & \cdots & 0 & 0 & 0 \\
\frac{1}{4} V_{1} & \frac{6}{8} V_{2} & \frac{1}{8} V_{3} & 0 & \cdots & 0 & 0 & 0 \\
0 & \frac{1}{8} V_{2} & \frac{6}{8} V_{3} & \frac{1}{8} V_{4} & \cdots & 0 & 0 & 0 \\
\vdots & \vdots & \vdots & \vdots & \ddots & \vdots & \vdots & \vdots \\
0 & 0 & 0 & 0 & \cdots & \frac{1}{8} V_{N-2} & \frac{6}{8} V_{N-1} & \frac{1}{4} V_{N} \\
0 & 0 & 0 & 0 & \cdots & 0 & \frac{1}{8} V_{N-1} & \frac{3}{4} V_{N}
\end{array}\right) \text {, }
$$

With this modification, we keep the second order spatial accuracy of the original control volume method as shown by Eq. 18, while we now have access to $c_{1}$ since $V_{1} \neq 0$. Furthermore, if we sum all the equations of each shell, we get,

$$
\sum_{i=1}^{N} V_{i} \frac{\partial c_{i}}{\partial t}+j r_{N}^{2}=0
$$

This satisfies the mass conservation condition exactly.

Non-uniform Grid Spacing.--Since the boundary of each control volume is either at the center of the particle, surface of the particle, or the midpoint of two nodes, we can still approximate the variable $c$ and its derivative at such a boundary in second order with only two nearby points. This largely reduces the computation complexity and the implementation difficulty of moving the above method from uniform mesh to non-uniform mesh compared to other numerical methods such as finite volume or finite difference.

The control volume discretized formula for non-uniform mesh grids can be written as the following,

$$
\begin{aligned}
\frac{\partial}{\partial t}( & \left.\frac{1}{8} V_{i-1} c_{i-1}+\frac{6}{8} V_{i} c_{i}+\frac{1}{8} V_{i+1} c_{i+1}\right) \\
= & D\left(\frac{c_{i+1}+c_{i}}{2}\right)\left(\frac{r_{i+1}+r_{i}}{2}\right)^{2} \frac{c_{i+1}-c_{i}}{r_{i+1}-r_{i}} \\
& -D\left(\frac{c_{i}+c_{i-1}}{2}\right)\left(\frac{r_{i}+r_{i-1}}{2}\right)^{2} \frac{c_{i}-c_{i-1}}{r_{i}-r_{i-1}} .
\end{aligned}
$$

where $V_{i}$ here is the volume of a small control volume $\left[\frac{r_{i-1}+r_{i}}{2}, \frac{r_{i}+r_{i+1}}{2}\right]$ around the grid point $r_{i}$.

Time domain discretization.- We have now introduced two different methods to discretize the spatial coordinate of the PDE system in Eq. 1. With the differential Eq. 6 and Eq. 19, now we also need to seek for a time domain numerical discretization method to solve this problem. In this section, we first prove the systems Eq. 6 and Eq. 19 are both ordinary differential equations, instead of differential algebraic equations, as the former can be solved much more easily. Then we derive the formula for the implicit time solver we used in solving both ordinary differential equation systems, the Crank-Nicolson method.

Proof of ODE systems.-In order to prove the differential Eq. 6 and Eq. 19 are both ordinary differential equations, it is sufficient to prove the mass matrices $\mathbf{M}_{f}$ and $\mathbf{M}_{c}$ are both nonsingular. The only assumption we make here is that the volume $V_{i}$ is not zero for each $i$. The mass matrix $\mathbf{M}_{f}$ is a diagonal matrix, with $V_{i} \neq 0$ on each diagonal entry by our assumption. Then the statement that $\mathbf{M}_{f}$ is nonsingular follows immediately.

To prove that the mass matrix $\mathbf{M}_{c}$ is nonsingular, we can write $\mathbf{M}_{c}$ as a product of two matrices $\mathbf{M}_{1}$ and $\mathbf{M}_{2}$,

$$
\begin{aligned}
\mathbf{M}_{c}= & \left(\begin{array}{cccccccc}
\frac{3}{4} & \frac{1}{8} & 0 & 0 & \cdots & 0 & 0 & 0 \\
\frac{1}{4} & \frac{6}{8} & \frac{1}{8} & 0 & \cdots & 0 & 0 & 0 \\
0 & \frac{1}{8} & \frac{6}{8} & \frac{1}{8} & \cdots & 0 & 0 & 0 \\
\vdots & \vdots & \vdots & \vdots & \ddots & \vdots & \vdots & \vdots \\
0 & 0 & 0 & 0 & \cdots & \frac{1}{8} & \frac{6}{8} & \frac{1}{4} \\
0 & 0 & 0 & 0 & \cdots & 0 & \frac{1}{8} & \frac{3}{4}
\end{array}\right) \\
& \times\left(\begin{array}{cccccc}
V_{1} & 0 & \cdots & 0 & 0 \\
0 & V_{2} & \cdots & 0 & 0 \\
\vdots & \vdots & \ddots & \vdots & \vdots \\
0 & 0 & \cdots & V_{N-1} & 0 \\
0 & 0 & \cdots & 0 & V_{N}
\end{array}\right)=\mathbf{M}_{1} \mathbf{M}_{2} .
\end{aligned}
$$

$\mathbf{M}_{2}$ is nonsingular by the same proof that $\mathbf{M}_{f}$ is nonsingular. By Gershgorin's circle theorem, ${ }^{24}$ all eigenvalues of the matrix $\mathbf{M}_{1}$ are located within the circle centered at $\frac{6}{8}$ with radius $\frac{3}{8}$ on the complex plane. Therefore, 0 is not an eigenvalue to the matrix $\mathbf{M}_{1}$, and $\mathbf{M}_{1}$ is also a nonsingular matrix. It follows that the product of $\mathbf{M}_{1}$ and $\mathbf{M}_{2}$, $\mathbf{M}_{c}$, is also nonsingular. This finishes the proof that the discretized system Eq. 19 is an ODE system.

The Crank-Nicolson method.-The Crank-Nicolson method is a combination of the forward and backward Euler's methods that is used for solving ordinary differential equations with second order accuracy in the time discretization. It is widely used to time-integrate the diffusion equation in stable finite difference schemes. ${ }^{25}$ The basic idea involves centered differencing, similar to the control volume method developed above for the spatial integration. Given an initial concentration profile $\mathbf{c}^{t}$ and the time step size $\Delta t$, the prediction of concentration profile $\mathbf{c}^{t+\Delta t}$ at time $t+\Delta t$ satisfies,

$$
\mathbf{M} \frac{\mathbf{c}^{t+\Delta t}-\mathbf{c}^{t}}{\Delta t}=\frac{1}{2}\left(\mathbf{f}\left(\mathbf{c}^{t+\Delta t}\right)+\mathbf{f}\left(\mathbf{c}^{t}\right)\right),
$$

where $\mathbf{M}$ is the corresponding mass matrix.

It is equivalent to rewrite our problem in the following way. For each time step, we need to solve for $\mathbf{c}^{t+\Delta t}$ such that,

$$
\mathbf{g}\left(\mathbf{c}^{t+\Delta t}\right)=\mathbf{M} \frac{\mathbf{c}^{t+\Delta t}-\mathbf{c}^{t}}{\Delta t}-\frac{1}{2}\left(\mathbf{f}\left(\mathbf{c}^{t+\Delta t}\right)+\mathbf{f}\left(\mathbf{c}^{t}\right)\right)=0 .
$$


Table II. Parameter settings for numerical experiments.

\begin{tabular}{lccc}
\multicolumn{1}{c}{ Parameter Name } & Notation & Value & Unit \\
\hline Particle Radius & $R_{p}$ & $5 \times 10^{-6}$ & $\mathrm{~m}$ \\
Surface Flux & $j$ & $-5.35 \times 10^{-5}$ & $\mathrm{~mol} / \mathrm{m}^{2} / \mathrm{s}$ \\
Initial Concentration & $C_{0}$ & $2 \times 10^{4}$ & $\mathrm{~mol} / \mathrm{m}^{3}$ \\
Max Concentration & $C_{m}$ & $4.665 \times 10^{4}$ & $\mathrm{~mol} / \mathrm{m}^{3}$
\end{tabular}

To solve such a nonlinear algebraic system we employ Newton's method with an initial guess $\mathbf{c}^{t+\Delta t}=\mathbf{c}^{t}$. We can reduce the computation cost by taking advantage of the fact that the Jacobian matrix of function $\mathbf{g}$ with respect to $\mathbf{c}^{t+\Delta t}$ can be obtained analytically, ${ }^{26}$ thereby avoiding the need to calculate the Jacobians numerically in each iteration. When the diffusion coefficient $D$ is a constant, the vector function $\mathbf{f}$ is then linear to the variable $\mathbf{c}^{t+\Delta t}$. Therefore, it takes only one step to reach the solution.

\section{Numerical Results}

In this section we will show the results from numerical experiments for both the finite volume and control volume discretization methods coupled to the Crank-Nicolson solver. The numerical convergence order in both space and time coordinates will be demonstrated. We will also discuss the effects of the grid point locations on the numerical error, from which we may see that by optimizing the grid point locations for the diffusion coefficient function and parameter set we use here, we can considerably reduce the number of grid points while maintaining the same or even achieving higher accuracy.

In our numerical simulation, we select a parameter set that is typical for a lithium ion battery cathode active material. We use the diffusion coefficient function shown in Eq. 4, and the choice of particle radius, surface flux and initial concentration shown in Table II. The surface flux value, $j$, in Table II corresponds to a C-rate of about $4.3 \mathrm{C}$ (corresponding to a full discharge in about $14 \mathrm{~min}$ ), a rate that is reasonable for a PHEV vehicle battery.

A typical concentration distribution profile during the intercalation of lithium is shown in Fig. 3.

Error order analysis. - As demonstrated analytically in the previous sections, we expect second order accuracy in space and time for both the finite volume and the control volume discretization coupling to the Crank-Nicolson method.

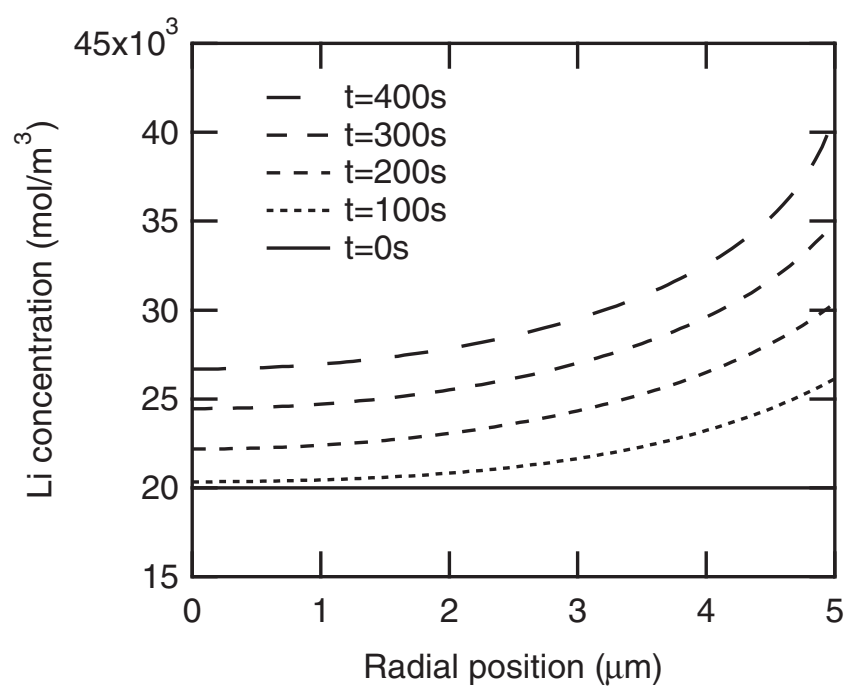

Figure 3. Concentration distribution within the spherical particle for a fixed surface flux, the parameters given in Table II, and a time step size of 5 seconds.

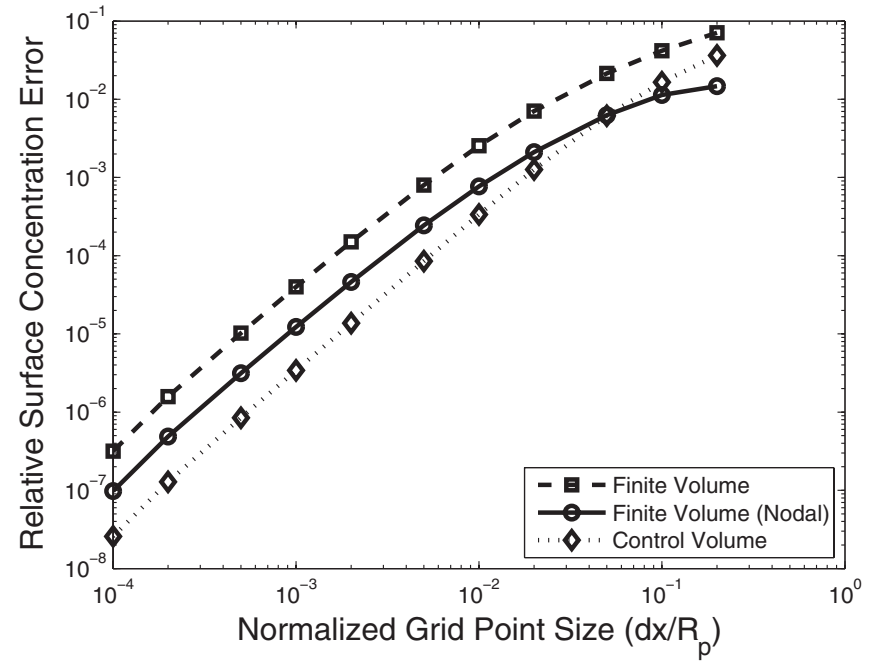

Figure 4. Plot showing the relative error convergence order of three numerical schemes in the spatial coordinate. The curve of finite volume method with extrapolation method Eq. 7 is shown by the dash line with square marker, and the solid line with circle marker represents the finite volume method with extrapolation method Eq. 9. The dot curve with diamond marker represents the control volume method. The relative error is defined as the error of surface concentration over the reference surface concentration. The total simulation time is 400 second with a time step of 5 seconds.

Since we are mostly interested in the surface concentration in our simulation, we define the error in terms of the accuracy of the surface concentration at the end of our simulation. We use the solution from a very fine grid mesh (50001 points) as our reference solution in the error convergence test.

It is clear from Fig. 4 that the finite volume method with two different extrapolations and the control volume method are in the second order as expected. However, for a fixed number of node points, the control volume method is more accurate than the finite volume method by a factor of about 10 . This shows one of the advantages of using the control volume method in the simulation.

For the time dimension, similar to the way we conduct the spatial error convergence test, we fixed the mesh size to be 101 uniform grid points and then varied the time step sizes. In addition, we took the solution with very small time step size (0.002 s per step) and the same mesh (101 grid points) as our reference solution.

As shown in Fig. 5, the slope of the log-log plot is 2, which indicates that for the given mesh size, all three numerical methods are second order accurate in time, which is consistent with our previous derivation of the Crank-Nicolson method.

Effects of grid point positions. - In the previous discussions of different numerical methods, we worked only with a uniform mesh within the spatial domain. Due to the physics of the problem, a Neumann boundary condition is required, such that the concentration in the region closest to the particle surface should change more rapidly than the region closest to the center. Furthermore, we are particularly interested in the surface concentration because of its use in other equations in our battery model. Therefore, it may be a good idea to use a non-uniform spatial mesh with more grid points closest to the surface, in order to achieve a better accuracy and/or a shorter simulation time.

Indeed, we find that the locations of the grid points have a significant influence on the numerical accuracy. Instead of using a constant flux, now we apply a varying flux by simulating a driving cycle. The driving cycle is composed of a series of surface fluxes, each applied for a duration of 1 second, and represents a real-world load profile applied in a vehicle application. The drive cycle we use consists of a period of city driving with a relatively low average load, followed by a period of highway driving with a higher average load, followed by a second period of city driving. 


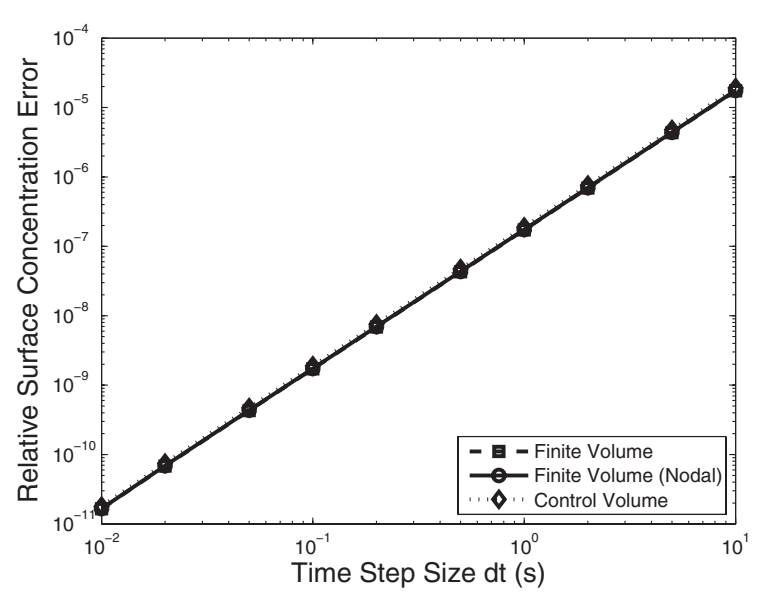

Figure 5. Plot of the relative error convergence order of three numerical schemes in the time coordinate. The curve of the finite volume method with extrapolation method Eq. 7 is shown by the dash line with the square marker, and the solid line with the circle marker represents the finite volume method with extrapolation method Eq. 9. The dot curve with diamond marker represents the control volume method. The relative error is defined as the error of surface concentration over the reference surface concentration. The total simulation time is 400 second with a 101-uniform-grid-point mesh.

Results are shown in Fig. 6, and were obtained using the same parameter values as in Tables I and II (with the exception of the timevarying surface flux). Figure 6(a) provides surface concentrations calculated with 501 uniform, 21 uniform, and 21 non-uniform grid points. The slope of the surface concentration vs. time is a rough indicator of the average load on the particle. The inset in Fig. 6(a) shows that 21 nonuniform grid points can provide a surface concentration that is much closer to the converged solution (with 501 uniform grid points) than 21 uniform mesh points. Indeed, the root-mean-square errors relative to the 501 uniform grid point solution are 4.48 and $55.73 \mathrm{~mol} / \mathrm{m}^{3}$ for the nonuniform and uniform cases, respectively. This shows that with 21 grid points, if we increase the density near the surface, we can achieve more than 10 times higher accuracy (lower RMS error) in the surface concentration than with the same number of uniform mesh grids. From a different point of view, 138 uniform grid points are required to achieve the same RMS error in the surface concentration as the 21 nonuniform grid points, demonstrating a $6.57 \mathrm{x}$ reduction in the number of points required for a given accuracy with the drive cycle and parameter set we use here. The nonuniform mesh point locations are given by,

$$
\vec{x}=\frac{\operatorname{logspace}(0, \mathrm{a}, 10)-1}{10^{a}-1},
$$

where logspace(.) is a function in MATLAB that provides logarithmically equally spaced points (in base 10) and $a$ is some negative number we varied from 0 (uniform grid in this case) to 4 . The form of Eq. 26 was chosen merely because it conveniently distributes most of the grid points near the particle surface, and is not the result of an optimization or systematic study of grid point placement.

Achieving an accuracy in the equilibrium potential of the $\mathrm{Li}\left(\mathrm{Ni}_{1 / 3} \mathrm{Mn}_{1 / 3} \mathrm{Co}_{1 / 3}\right) \mathrm{O}_{2}$ material in reference 22 of $1.0 \mathrm{mV}$ requires
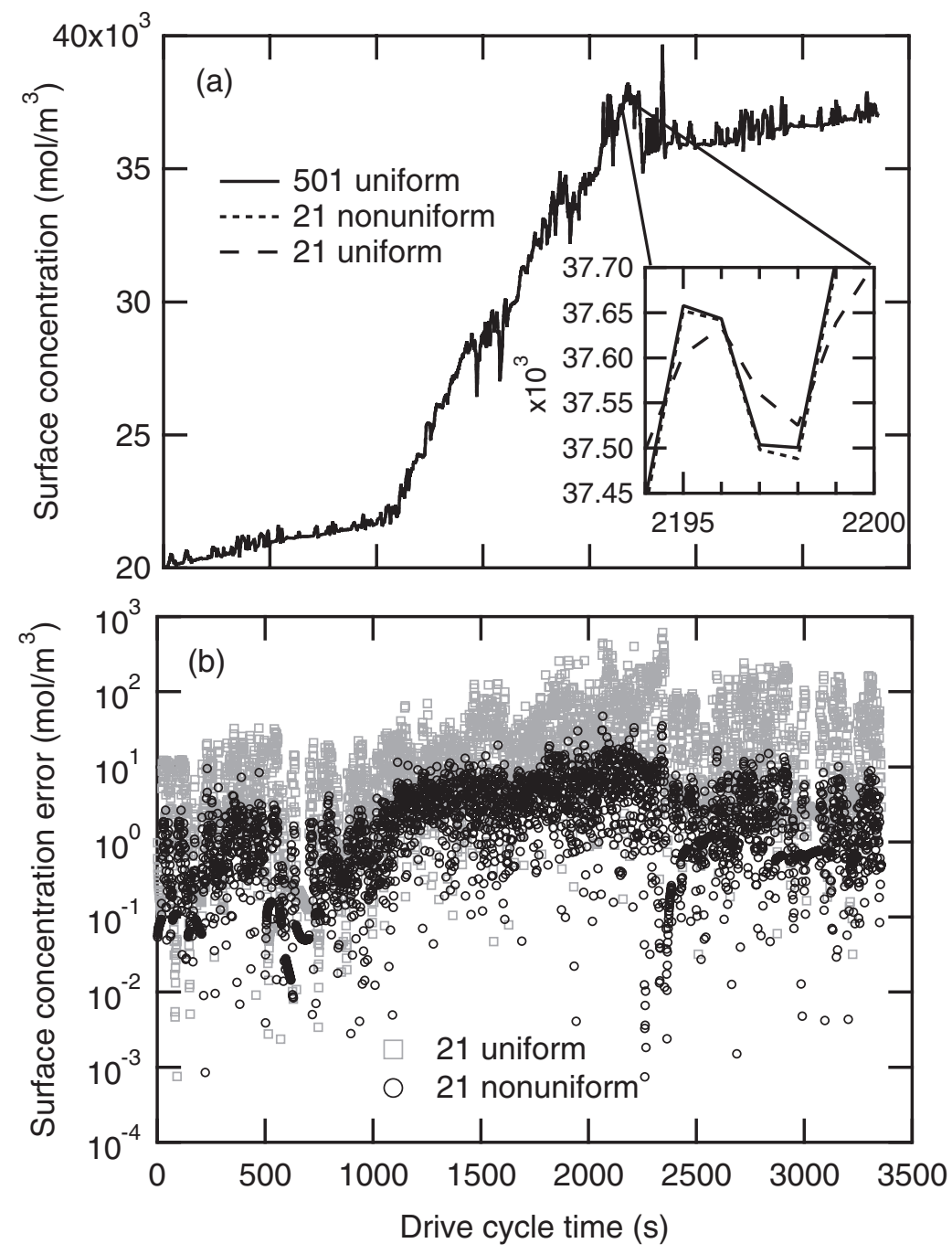

Figure 6. The surface concentration throughout a drive cycle with 501 uniform grids (the reference solution, black solid curve), 21 non-uniform grids (narrow dash curve), and 21 uniform grids (wide dash curve) are shown in the top subfigure (a), while the corresponding absolute errors in surface concentrations over time with 21 uniform grids (gray squares) and 21 non-uniform grids (black circles) are shown in the bottom subfigure (b). We may see the performance from 21 non-uniform grid points is significantly better than the outcome from 21 uniform grids. The RMS (short for "root mean square") error in the surface concentration for the uniform grid is $55.73 \mathrm{~mol} / \mathrm{m}^{3}$ while the RMS error for the non-uniform grid is only $4.48 \mathrm{~mol} / \mathrm{m}^{3}$. We chose the parameter $a=-1.5$ in Eq. 26 for the non-uniform grid. 
an accuracy in the surface concentration of about $10 \mathrm{~mol} / \mathrm{m}^{3}$ (for all but the tail region below about $3.6 \mathrm{~V}$ vs. Li). As accurate battery simulations require highly accurate equilibrium potential values, achieving an RMS error in the surface concentration that is below $10 \mathrm{~mol} / \mathrm{m}^{3}$ is desirable. The RMS error in the surface concentration of $4.48 \mathrm{~mol} / \mathrm{m}^{3}$ for 21 non-uniform grid points is below this target, while 21 nonuniform grid points give an RMS error in the surface concentration $\left(55.73 \mathrm{~mol} / \mathrm{m}^{3}\right)$ that is far above that bound. Fig. 6(b) shows that while the RMS error of the surface concentration with 21 non-uniform grid points may be below $10 \mathrm{~mol} / \mathrm{m}^{3}$, there are points in the drive cycle when the error is significantly higher, demonstrating the need for a battery modeler to carefully select model parameters (including number of mesh points and mesh point spacing) that give an accuracy suitable for the modeling purpose.

The dramatic reduction in the number of required points while maintaining a high accuracy provides inspiration to optimize the grid point location based on the particle size, diffusion coefficient (including functional forms that describe the dependence of the diffusion coefficient on concentration), and input flux profile. ${ }^{21}$ The goal is to allocate mesh points within the spatial domain while maintaining the same surface concentration solution from the coarse, nonuniform grid and the very fine, uniform grid. With this coarse mesh, we may significantly reduce the simulation time of this spherical diffusion process.

\section{Conclusions}

By carefully comparing the finite volume and control volume methods as applied to the $1 \mathrm{D}$, non-linear, spherical diffusion problem, we have shown that the advantages of the control volume method include directly obtaining the surface concentration rather than obtaining a volume-averaged concentration, a higher solution accuracy for a given number of node points, and a straightforward extension of the method to non-uniform grid spacing that can significantly reduce computational time by selectively placing grid points where concentrations gradients are highest.

We have quantified the errors in the surface concentration that come from both uniform and non-uniform meshes and compared the errors with an accurate solution. Our results underscore the importance of understanding the impact of the numerical solution technique for the solid transport process in order to achieve an accuracy appropriate for the modeling purpose, as the surface concentration (or activity) determines both the equilibrium potential and exchange current density typically used in battery models. The control volume method can also be extended to solving accurately more complex battery models, ${ }^{15}$ such as the fourth-order, nonlinear Cahn-Hilliard reaction model, ${ }^{1}$ where the surface activity also depends on concentration gradients and elastic stresses.

\section{Acknowledgments}

This material is based upon work supported by the National Science Foundation Graduate Research Fellowship under Grant No. 1122374. The information, data, or work presented herein was funded in part by the Advanced Research Projects Agency-Energy (ARPA-E), U.S. Department of Energy, under Award Number DE-AR0000278.

\section{References}

1. M. Z. Bazant, Accounts of Chemical Research, 46, 1144 (2013).

2. P. Albertus, J. Christensen, and J. Newman, Journal of The Electrochemical Society, 156, A606 (2009).

3. M. Doyle, T. F. Fuller, and J. Newman, Journal of The Electrochemical Society, 140 1526 (1993).

4. T. F. Fuller, M. Doyle, and J. Newman, Journal of The Electrochemical Society, 141, 1 (1994).

5. K. Thomas, J. Newman, and R. Darling, Advances in lithium-ion batteries, 345 (2002).

6. T. R. Ferguson and M. Z. Bazant, Journal of The Electrochemical Society, 159, A1967 (2012).

7. V. Srinivasan and J. Newman, Journal of The Electrochemical Society, 151, A1517 (2004).

8. S. Dargaville and T. Farrell, Journal of The Electrochemical Society, 157, A830 (2010).

9. G. Singh, D. Burch, and M. Z. Bazant, Electrochimica Acta, 53, 7599 (2008)

10. P. Bai, D. Cogswell, and M. Z. Bazant, Nano Letters, 11, 4890 (2011).

11. D. A. Cogswell and M. Z. Bazant, ACS nano, 6, 2215 (2012).

12. D. A. Cogswell and M. Z. Bazant, submitted (2013).

13. J. Song and M. Z. Bazant, Journal of The Electrochemical Society, 160, A15 (2013)

14. D. Burch and M. Z. Bazant, Nano Letters, 9, 3795(2009).

15. Y. Zeng and M. Z. Bazant, MRS Proceedings, 1542 (2013).

16. C. Wang, W. Gu, and B. Liaw, Journal of The Electrochemical Society, 145, 3407(1998)

17. V. R. Subramanian, V. D. Diwakar, and D. Tapriyal, Journal of The Electrochemical Society, 152, A2002 (2005).

18. S. Liu, Solid State Ionics, 177, 53(2006).

19. Q. Zhang and R. E. White, Journal of Power Sources, 165, 880 (2007)

20. K. Smith and C.-Y. Wang, Journal of Power Sources, 161, 628 (2006).

21. V. Ramadesigan, V. Boovaragavan, J. C. Pirkle, and V. R. Subramanian, Journal of The Electrochemical Society, 157, A854 (2010).

22. S.-L. Wu, W. Zhang, X. Song, A. K. Shukla, G. Liu, V. Battaglia, and V. Srinivasan, Journal of The Electrochemical Society, 159, A438 (2012).

23. M. Ó. Searcóid, Metric spaces (Springer, 2006).

24. S. A. Gershgorin, Bulletin de l'Académie des Sciences de l'URSS. Classe des sciences mathématiques et na, 749 (1931).

25. J. C. Strikwerda, Finite Difference Schemes and Partial Differential Equations, second edition ed. (SIAM, 2004).

26. K. T. Chu, Journal of Computational Physics, 228, 5526 (2009). 